\title{
RadSem: Semantic Annotation and Retrieval for Medical Images
}

\author{
Manuel Möller ${ }^{1}$, Sven Regel ${ }^{2}$, and Michael Sintek ${ }^{1}$ \\ 1 German Research Center for Artificial Intelligence (DFKI) GmbH \\ Kaiserslautern, Germany \\ manuel.moeller@dfki.de, michael.sintek@dfki.de \\ 2 Chemnitz University of Technology, Chemnitz, Germany \\ sven.regel@cs.tu-chemnitz.de
}

\begin{abstract}
We present a tool for semantic medical image annotation and retrieval. It leverages the MEDICO ontology which covers formal background information from various biomedical ontologies such as the Foundational Model of Anatomy (FMA), terminologies like ICD-10 and RadLex and covers various aspects of clinical procedures. This ontology is used during several steps of annotation and retrieval: (1) We developed an ontology-driven metadata extractor for the medical image format DICOM. Its output contains, e.g., person name, age, image acquisition parameters, body region, etc. (2) The output from (1) is used to simplify the manual annotation by providing intuitive visualizations and to provide a preselected subset of annotation concepts. Furthermore, the extracted metadata is linked together with anatomical annotations and clinical findings to generate a unified view of a patient's medical history. (3) On the search side we perform query expansion based on the structure of the medical ontologies. (4) Our ontology for clinical data management allows us to link and combine patients, medical images and annotations together in a comprehensive result list. (5) The medical annotations are further extended by links to external sources like Wikipedia to provide additional information 1
\end{abstract}

\section{Introduction}

Advances in medical imaging have enormously increased the volume of digital images produced in clinical practice. At the same time, modern hospital information systems have also become more complex. Radiological findings are kept separately from images which, in turn, are kept separately from patient accounting and billing information.

Currently, these systems are more or less isolated from each other and do not allow queries to span across these systems. Thus it has become challenging for clinicians to query for and retrieve relevant historical data due to the volume

${ }^{1}$ This research has been supported in part by the THESEUS Program in the MEDICO Project, which is funded by the German Federal Ministry of Economics and Technology under the grant number 01MQ07016. The responsibility for this publication lies with the authors. 
of information as well as the complexity of information systems. In particular, historical patient images would be useful for analyzing images of a current examination since they help in understanding any progression of pathologies or development of recent abnormalities. Today, such images can only be retrieved by attributes stored in the DICOM ${ }^{2}$ headers of the images such as patient name, age or gender. However, these attributes do not contain any information about the anatomy or disease associated with the image. Hence, radiologists are often overwhelmed with irrelevant images not connected with the current examination or - the other extreme - are unable to retrieve any similar case.

To overcome these limitations our system uses the Semantic Web standards OWL [9] and RDF [7] as a common umbrella to represent domain knowledge and annotations in the same formalism. We have developed the MEDICO Ontology Hierarchy (see Fig. 1) which models various aspects of clinical data management and medical background knowledge. Our rationale was to reuse background knowledge represented in formal ontologies such as the Foundational Model of Anatomy ontology (FMA) [19] and terminologies like RadLex [8] and the International Classification of Diseases version 10 (ICD-10) 3 Wherever possible we used existing translations to OWL. For the ICD-10 we implemented our own technique which is described in Sect. 3.2. These ontologies were integrated into our hierarchy to provide background knowledge about the medical domain during annotation and retrieval.

On the search side we leverage the structural information in the ontologies to allow multilingual search and to perform query expansion to retrieve images which are annotated with semantically similar concepts.

Semantic Web technologies allows us to reuse highly qualified medical domain knowledge for annotation and query expansion without being medical experts ourselves. Storing the medical annotations as instances of well defined OWL classes - rather than in a proprietary relational database - fosters an open interchange of this data with other applications and makes them easily available for other research goals, such as clinical data mining. The fusion of medical annotations with automatically extracted patient and image metadata in the same representation formalism provides the basis for new intuitive visualizations.

The research on semantic annotation and retrieval described in this paper is part of a broader effort to understand the semantic of medical images in the THESEUS MEDICO 12 project.

\section{Related Work}

The need for representing high-level annotations of medical images on an abstract level has been emphasized in various publications in recent years, e.g., in $13,23,14$.

Biomedical ontologies and terminologies received high attention in the last decade and provide promising technologies. 3] evaluated popular large scale

\footnotetext{
${ }^{2}$ Digital Imaging and Communication standard, http://medical.nema.org/
}

3 http://www . who.int/classifications/apps/icd/icd10online 
ontologies such as SNOMED, FMA, and Gene Ontology and stated that "ontologies play an important role in biomedical research through a variety of applications". Only recently, 10] presented an approach for integrating FMA and RadLex as FMA-RadLex to compile a robust application ontology. Besides efforts combining ontologies and radiological reports (e.g., 15]), other approaches using ontologies in medical image processing have been proposed [18, 22.

The work in 20, is similar to our research in annotating medical images with ontology-based semantics and the use of context for faster annotation. However, their notion of context is primarily oriented on anatomy than diseases.

\section{Ontological Modeling}

Fig. 1 gives an overview of the general structure of the MEDICO Ontology Hierarchy. The following paragraphs describe the different parts of the hierarchy in detail as well as their interrelations and the design rationales. We follow Gruber's definition 5] for the term ontology that "an ontology is a

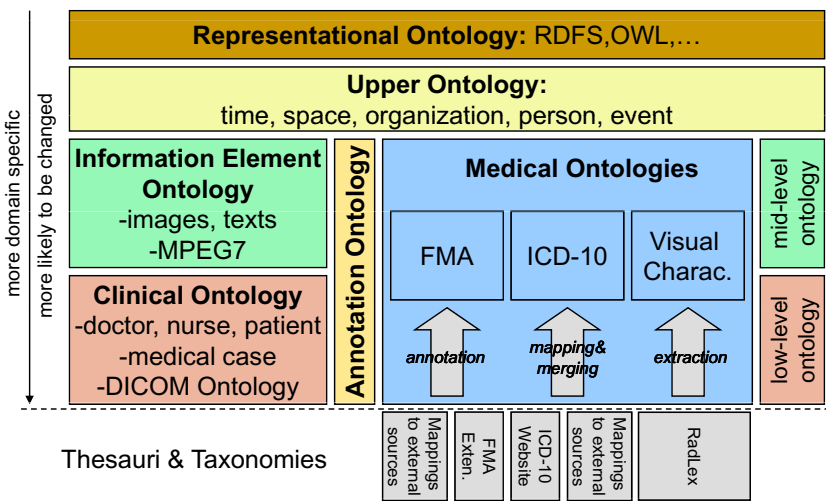

Fig. 1. MEDICO Ontology Hierarchy formal specification of a (shared) conceptualization." An OWL model of this ontology hierarchy can be browsed online 4

Ontologies are usually structured in various layers or levels, based on the assumption that those at higher levels are more stable, shared among more people, and thus change less often than those at lower levels. Following [21, we distinguish representational ontologies, upper-level ontologies, mid-level ontologies, and low-level or domain ontologies.

\subsection{Medical Ontologies}

In the medical domain, large amounts of knowledge are already formulated in ontologies like the FMA. Therefore we decided to reuse those sources and opted for a mapping to/merging of those ontologies and thesauri each covering different dimensions of image annotation.

We differentiate between three different aspects or dimensions of medical annotation. For anatomy we use the FMA. The concepts for the visual manifestation of an anatomical entity on an image is derived from the modifier and

\footnotetext{
4 http://www.dfki.uni-kl.de/ moeller/ontologies/medico-browser
} 
imaging observation characteristic sub-trees of RadLex. We consider the disease aspect as the interpretation of the combination of the previous two. Here we use the ICD-10.

\subsection{ICD-10}

At the time of writing, ICD-10 was not available in any format following the Semantic Web standards. Due to our decision to use OWL and RDF in MEDICO, we generated a light-weight ICD-10 model in OWL which mainly reflects the terminological structure.

From the semistructured online version of the terminology, we have created a simple model using the OWL API 2]. It reflects the hierarchy as defined in the ICD-10 manual [4. We modeled this hierarchy on the class level while instances are considered to reflect concrete diseases of concrete patients. The whole OWL model contains 11,290 classes and has a maximum depth of 5 .

\subsection{Annotation Ontology}

The Annotation Ontology is used to connect a given medical document-be it image or text - with the concepts in the ontologies described in the previous sections. The diagram in Fig. 2 illustrates the ontology we created to generate annotations. (Ovals depict properties, rectangles classes.)

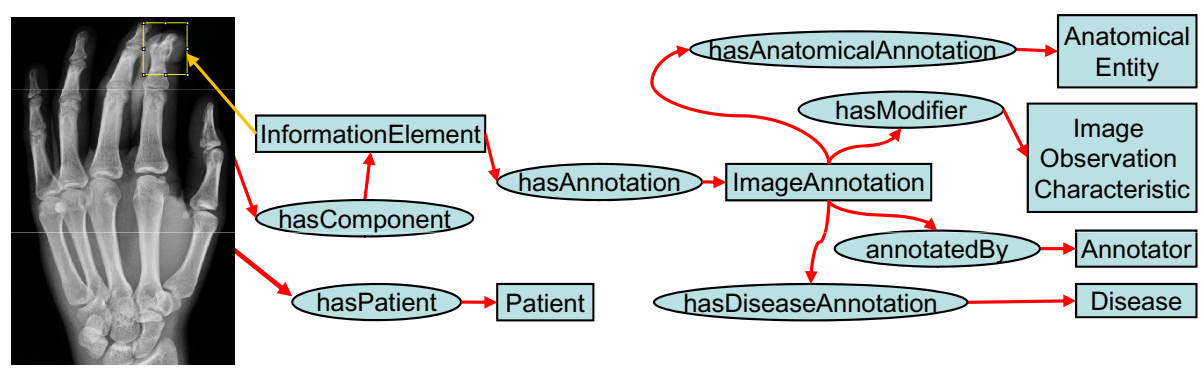

Fig. 2. Annotation Ontology

The image is connected to the patient via the hasPatient property. Additionally, we add annotations to the study and series data the image belongs to. For the sake of clarity we omitted these links in the illustration.

The image itself is decomposed into InformationElement instances with each of these instances being connected via the property hasAnnotation to an ImageAnnotation instance. InformationElement subsumes arbitrary documents (text, images, 3D volumes, ...) as well as parts or regions of them.

For each ImageAnnotation we store information on the annotation provenance. Annotator can either be a Physician instance for manual annotations by a medical expert or an instance of Classifier for automatically generated image annotations which we plan to integrate in the future. 
Finally, ImageAnnotations can be further qualified by adding instances of VisualModifier. Here we reuse the existing RadLex vocabulary enriched by terms from our own studies on radiology reports to identify further terms that are common in the clinical practice but not yet part of RadLex. These qualifiers comprise attributes to describe alterations from normal and healthy features like bulky or compressed.

\subsection{Mapping from DICOM Body Regions to FMA Body Regions}

Standardized formats such as DICOM have been developed for digitally representing the acquired images from the various modalities. Apart from the image pixels, a DICOM image also contains a header, which is used to store certain patient information such as name, gender, demographics, etc.

In order to create a deep interconnection between data from the DICOM metadata and the medical domain knowledge, we manually defined mappings for the 20 allowed values of the DICOM tag Body Part Examined to FMA concepts. We are aware of former studies like [6] which showed that the value of this field is in practice neither mandatory nor always correct. But in our opinion it would be wrong to completely ignore this information since it is correct in most of the cases. Instead we propose to put a bias on their value, signifying that their value is not absolutely reliable. In fact this is also the case with all results from automatic object recognition algorithms we plan to incorporate.

If there is no body region set in the DICOM header, we offer all concepts from the FMA for the anatomical annotation. If the wrong body region was saved to the DICOM header, this becomes apparent by comparing the body region visualization and the anatomical structures on the image. In both cases the user can set the correct body region manually in the application.

In the manual annotation tool we use the body region information from the DICOM header to select only those concepts from the FMA which belong to the body region. This reduces the number of potential anatomical annotation concepts from approx. 80,000 down to a few hundred to a few thousand.

Another application in the area of visual object recognition of the body region annotations is described in 11: A Metadata Extraction Module retrieves the BodyRegion from the DICOM header. Based on the mapping, the FMA is used to retrieve a list of anatomical entities that could possibly be detected in this body region. This narrows down the search space of potential objects on the image significantly.

\subsection{Body Substance Annotations for FMA}

Each body substance has its own characteristic radiation attenuation spectrum which is typically measured in Hounsfield Units 5 On CT and X-ray images this manifests in specific pixel contrast ranges for the tissue types. We have modeled the class TissueType which currently subsumes classes for 13 different body

${ }^{5}$ The Hounsfield Scale is a quantitative scale describing radiodensity. 
substances like Bone, Blood, etc. each annotated with the respective Hounsfield range.

In a second step they were mapped manually to FMA concepts. We considered implementing generic heuristics which traverse particular relationships in the FMA to retrieve all related concepts belonging to a specific tissue type. But so far we did not discover any heuristic generic enough to treat organs and their sub-components the same way as blood vessels which belong to organs.

The snapshot in Fig. 3 of the FMA shows a part of the part_of sub-tree of the concept Liver. It clearly shows, that veins and arteries are also considered to be part of the liver and not the artery system. This also holds for the more specific properties systemic_part_of, regional_part_of

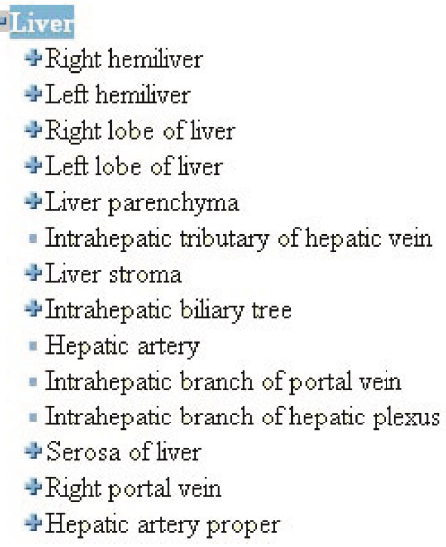

Fig. 3. part_of sub-tree of the Liver in the FMA and constitutional_part_of. We decided against the annotation of all constitutional parts of an organ with the same tissue type as their super class. The reason was that constitutional parts also comprise blood supply which has a different radiodensity.

\subsection{Mappings to External Biomedical Data Sources}

Here, our goal was to augment the annotations of search results with mappings to external data sources such as Wikipedia to provide additional information explaining the annotation concepts. The first step was to extract a list of all class labels and synonyms from the ontologies. Next, we iterated over this list and tried to identify the most relevant document in a corpus of potentially relevant web pages by performing a statistical keyword/phrase search. The corpus was indexed offline using the DynaQ API [1].

We searched for English labels and synonyms of the FMA in Wikipedia. In our first attempt we did a plain full text keyword search for labels and synonyms and created one mapping for the best match if there was one. This resulted in mappings for 86\% (84685 absolute, column keyword in Tab.1) of all FMA labels and synonyms. Prima facie this number is surprisingly high since most of the FMA concepts are very specific and we did not expect Wikipedia to cover them to such a high extent.

However, the quality of these mappings turned out to be very limited. We imposed a systematic evaluation by picking a random set of 100 mappings and checking each of them manually. We distinguished between (1) perfect match where the Wikipedia article is covering exactly the FMA concept; (2) a "useful" match meaning that the Wikipedia article covers (a) an anatomical part of the search label, (b) the FMA label is a part of the concept covered in the Wikipedia article, or (c) the Wikipedia article deals with a direct sub- or 
Table 1. Precision for different mapping types

\begin{tabular}{|l|c|c|c|c|}
\hline search method & keyword & phrase & phrase AND "Anatomy" & phrase in title \\
\hline perfect match & 33 & 30 & 26.7 & 64.7 \\
"useful" match & 33 & 21 & 18.7 & 15.7 \\
non-match & 34 & 49 & 54.6 & 19.6 \\
\hline
\end{tabular}

superclass of the FMA concept; and (3) the rest is regarded as non-match. It turned out that only $33 \%$ of the mappings were perfect matches. Subsequently, we switched to phrase-based search which lowered the number of mappings to 6.7\% (6 439 absolute, column phrase in Table 1). To overcome the problem of obviously irrelevant mappings we confined the results to articles containing the FMA label as a phrase and the keyword "Anatomy". This resulted in almost $50 \%$ wrong mappings (column phrase AND "Anatomy" in Tab. 1). By further restricting the search to pages which also contain the keyword "Anatomy" we tried to limit the results to articles of the target domain. However, this dropped the number of matches because "Anatomy" does not necessarily appear in all articles dealing with anatomical topics. Still this did not lower the number of irrelevant articles significantly. Finally we confined the search to the article titles which reduced the ratio of non-matches to less than $20 \%$ (column phrase in title in Table 1).

The additional restrictions cut down the overall number of mappings from 6439 to 4104 . We decided to trade precision for recall, provide less mappings and in turn avoid dissatisfaction due to irrelevant mappings.

\subsection{DICOM Ontology}

Since one of our main goals is to incorporate existing data based on established standards, the design of the clinical ontologies is to quite some extent aligned with the DICOM standard. This standard is a world wide established format used by hospitals to store the results of visual examinations (e.g., X-Ray, CT, PET, etc.) as well as patient and image metadata.

There are other efforts towards creating a DICOM ontology 6 but to our knowledge no OWL model has been released so far. Therefore we decided to model a MEDICO DICOM ontology. Our rationale was to keep closely with the DICOM standard in its version 3 from 2008. The reason was that many processes of clinical data management are use this structure and our intention is to support these processes instead of replacing them.

As discussed above (Sect. 3.4), the imaging equipment manufacturers do not always follow standards when it comes to storing imaging metadata. We also see this in our corpus. To cope with this fact, we decided on a hybrid methodology for our modeling. We combined a data-driven bottom-up with a top-down approach driven by the DICOM standard. We started by looking at the metadata available in the images of our corpus (see Sect. 5.1) and checked the standard for regulations regarding format, value ranges and meaning.

\footnotetext{
${ }^{6}$ For example within the caBIG project, http://cabig.nci.nih.gov/
} 
The DICOM standard organizes data elements in a simple hierarchy. In a clinical data management system each patient's images arranged in one or more Studies. A Study contains one or more Series which in turn contains one or more Image(s). Our modeling reflects this hierarchy and assigns properties only once depending on their domain. Each DICOM file has a comprehensive DICOM header which contains information about the patient, study and series it belongs to, date and time and several image acquisition parameters. In the following we introduce our approach by example.

Each metadata element in the DICOM standard has a fixed so called DICOM tag identifying it in the DICOM file header. To control the mapping process from DICOM metadata fields to properties in our ontology we annotated those properties with the respective tag using the annotation property dicomTag.

For example, the DICOM tag 0010,1010 always identifies the patients age for the current image/series, which we store in the datatype property age. Since DICOM specifies date and time formats differently from the XSD-based datatype properties in our ontology we have added the annotation property converter in order to control the conversion of each datatype property separately.

\section{Application}

Fig. 4 shows the annotation frontend of our application. Images are loaded and displayed using the API of the image processing program Image. 7 . Using this library images are segmented into regions of interest (ROI). Currently we support rectangular, ellipsoid and arbitrary polygons to confine these regions. These three types are modeled as different classes in our ontology. As discussed in Sect. 3.1. we distinguish between anatomy, image observation characteristic and disease which can be annotated separately for each ROI. To ease the task of finding appropriate annotations we use auto-completing combo-boxes. While typing in a search term, concept names with matching prefixes are shown in a drop down box and can be selected.

\subsection{DICOM Metadata Extractor}

Based on our modeling of the DICOM ontology we have extended the metadata extraction framework Aperture 8 with a metadata extractor for DICOM images. Controlled by the special annotation properties described in Sect. 3.7. it crawls file systems, web servers and WebDAV repositories and converts all available DICOM metadata to instances of the classes modeled in our ontology.

In fact, each DICOM image contains all information about patient, series and study which leads to a huge data redundancy considering volume datasets (i.e., a stack of images generated by a CT scan) which often contain more than 100 images. In such cases all images of a series are linked to the same instance of a patient in contrast to the redundant storage in the DICOM headers.

\footnotetext{
7 http://rsbweb.nih.gov/ij/

8 http://aperture.sourceforge.net/
} 


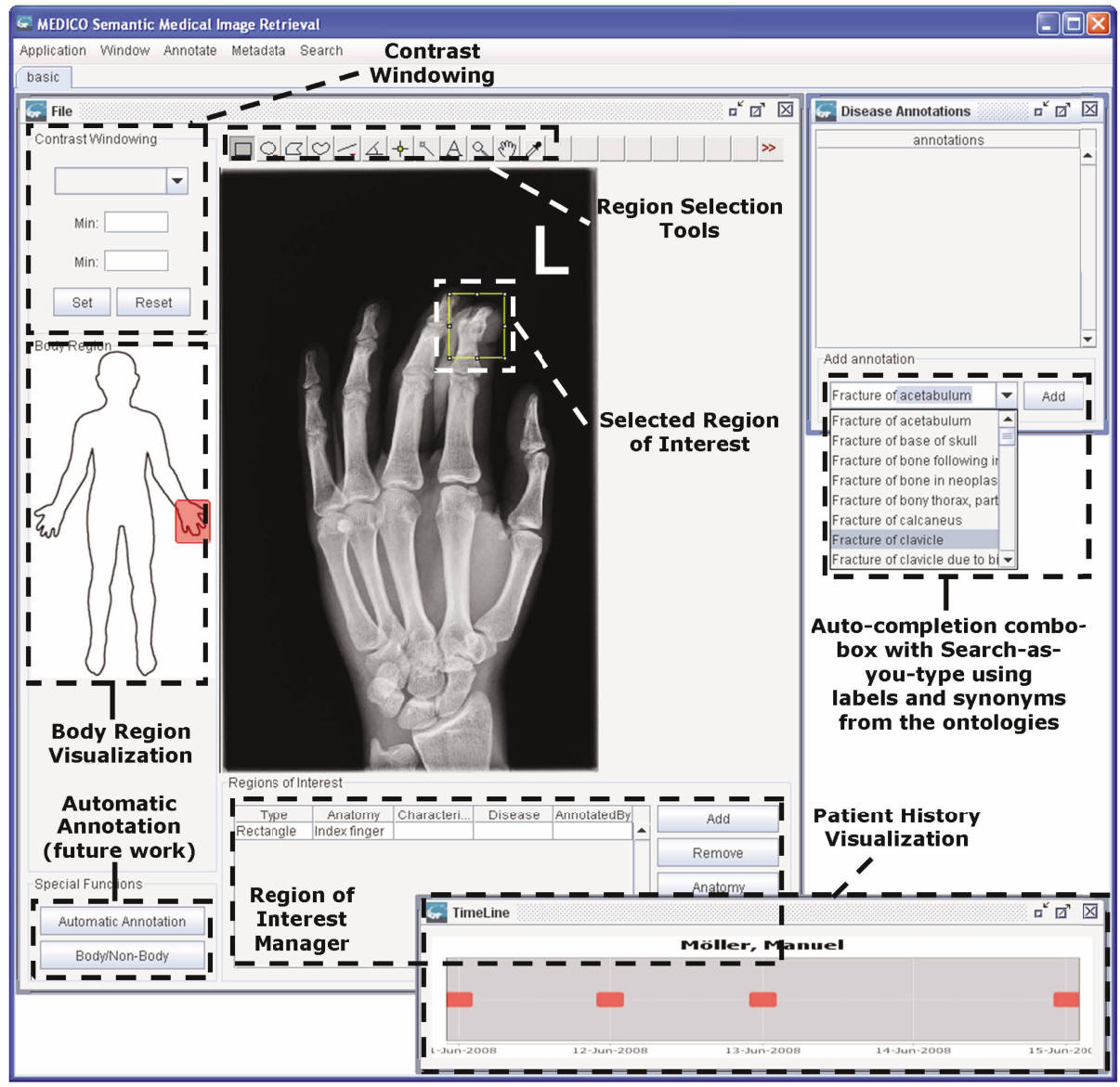

Fig. 4. Annotation Frontend

In our annotation application this metadata can be accessed during annotation and retrieval and is rendered using the presentation vocabulary Fresnel [16. Additionally, we have set up a separate faceted browsing application 9 using MIT's Longwell 10

\subsection{Annotation Interface}

Contrast Windowing: The medical background knowledge tells a radiologist that, e. g., he has to adjust the contrast range to 80-1000 if he wants to highlight bones in a CT scan. Likewise we offer the TissueType annotations introduced in Sect. 3.5 in the annotation tool to dynamically adjust the contrast range of the current image (see Fig. 4). This feature is included in many medical imaging

\footnotetext{
9 http://www.dfki.uni-kl.de/medico-longwell-2.5.5

10 http://simile.mit.edu/wiki/Longwell
} 


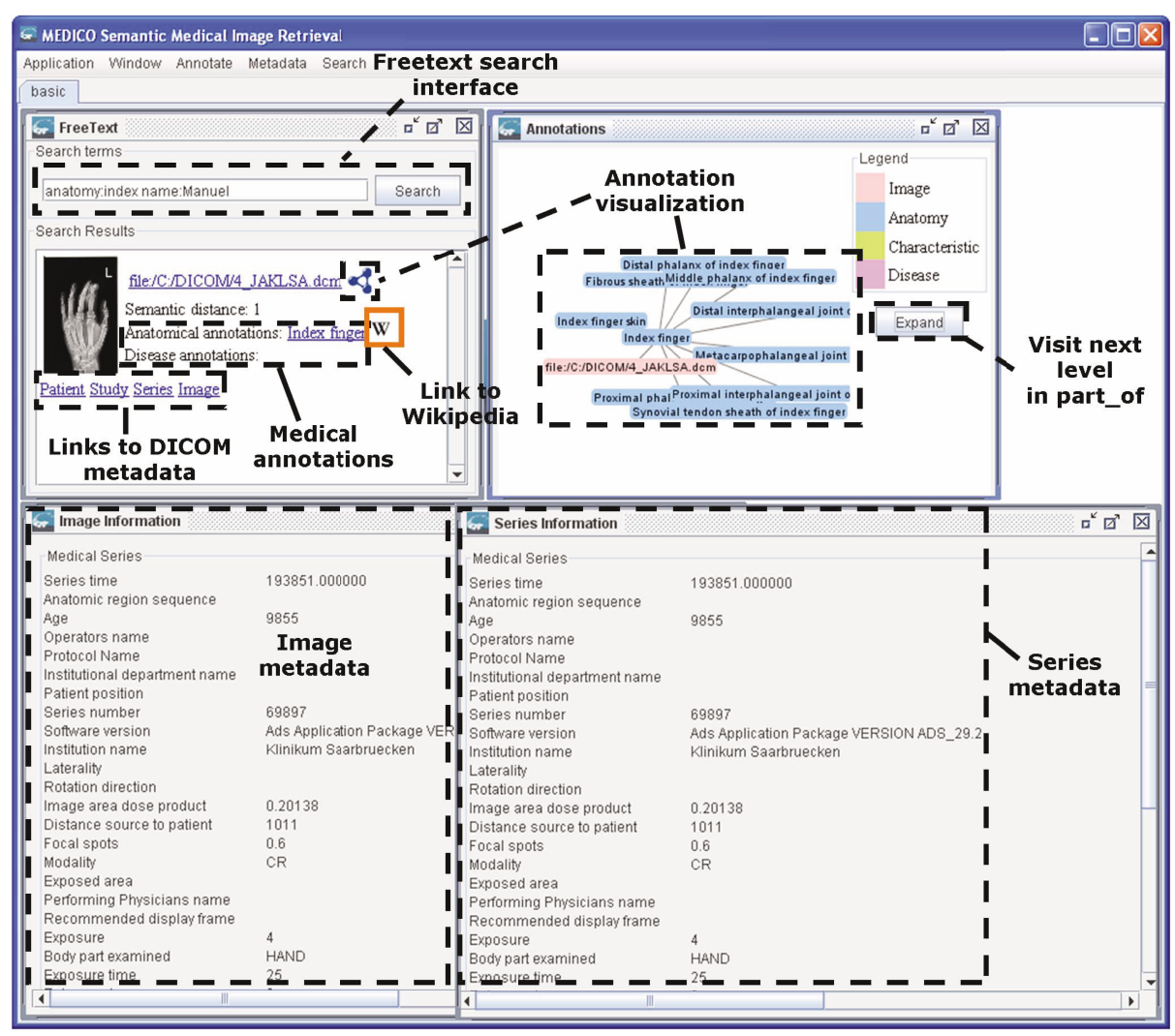

Fig. 5. Search Frontend

applications. But here we use one single abstract modeling of tissue types both for manual annotation as well as to control the preprocessing in automatic image annotation workflows, which is beyond the scope of this publication and further described in [1].

Body Region Visualization: The mapping between DICOM body region and FMA body region annotations as introduced in Sect. 3.4 is also used to provide a visualization of the body region from which the current image is taken. Upon loading an image, the body region metadata tag of the DICOM image is used to visualize the body region of the current image in the silhouette of a prototypical human (see Fig. 4).

Patient History Visualization: Medical diagnosis is largely dependent on the medical history of a patient. On this account we offer a visual timeline of previous radiological examinations. Each available image series is displayed as a box on a time axis in chronological order (see Fig. 4). This timeline is generated on the fly from the automatically extracted information from the 
DICOM headers and former manual annotations from the radiologists. Even without any manual annotations, the user immediately gets an overview about former visual examinations of the patient. Clicking on a series opens a window with all available information about this series and links to corresponding images.

\subsection{Search Interface}

In the Lucene 11 -like free text search, arbitrary queries can be created by specifying Lucene labels such as "anatomy:" and corresponding values like "Index".

This allows the creation of hybrid queries searching for manual medical annotations as well as specific metadata values as extracted from the DICOM metadata extractor (Sect. 4.1). For example, you can specify that the name of the patient has to match a certain substring, e.g., by adding "name:Manuel" to the query. The values of search labels such as "anatomy" and "disease" are mapped in the background to concepts in the medical ontologies.

The results contain a thumbnail, the full path of the image and all available annotations. By clicking on the RDF icon a separate frame is opened which visualizes all annotations. A click on the "Expand" button retrieves all child concepts of all annotated concepts and thus allows the exploration of the semantic "neighborhood".

Query Expansion: For searching we leverage the structural information in the medical ontologies. In the part-of hierarchy of the FMA there is a path from the concept Index finger (the concept used originally to annotate the image) to Index which was the search concept in the example in Fig. 5. Internally we use this information to compute a query expansion and translate it into a SPARQL 17. query. Using the FMA, a search for concept $X$ searches for the concept itself and all concepts which have a (transitive) link via the properties regional_part_of and constitutional_part_of to $X$. To rank the results, we compute the path length from the search concept to the concept used for annotation of the search results. Using ICD-10, we traverse the subClassOf property downwards.

Traversing the FMA recursively with SPARQL queries at runtime turned out to be way too slow. The FMA uses the equivalent of "multiple inheritance" in the part_of hierarchy. E.g., Heart is both part of Set of thoracic viscera and of Content of middle mediastinum. Thus we decided to materialize a spanning tree for the properties regional_part_of and constitutional_part_of starting from the concept Human body. By materializing the shortest distances to the root node of this closure, we are able to perform a query expansion with arbitrary search depth limit in two retrieval operations: The first query is used to retrieve the list of classes in the part_of spanning tree down to a certain depth limit and the second to retrieve the annotated images.

Of course we would prefer to find a solution that did not require to materialize closure and distance information, since this would save us a lot of memory. But at the time of writing, our system setup with SwiftOWLIM 12 version 3 on a

\footnotetext{
11 http://lucene.apache.org/

12 http://www.ontotext.com/owlim/
} 
quadcore machine with 32 GB RAM did not allow reasonable retrieval times without materialization.

Multilingual Annotation and Search: Another advantage of using existing ontologies such as the FMA is the availability of multilingual synonyms. Currently, we allow annotation and search using any synonym available in the FMA. English, Latin, French, German and Filipino are covered and the preferred 13 annotation language can be changed at runtime.

\section{Evaluation}

\subsection{Our Corpus}

Acquiring medical images for testing purposes is a big problem. Legal regulations enforce a very strict anonymization both for clinical patient records as well as any personal information in the DICOM headers. Because we wanted to test our extractor with real data and avoid systematic errors by testing only with values generated by anonymization algorithms, we added non-anonymized personal images to the corpus. All images and metadata shown on screenshots in this publication belong to examinations performed on one of the authors during the summer of 2008 .

\subsection{Qualitative Evaluation}

When it comes to the evaluation of systems using technologies from the Semantic Web, evaluation turns out to be problematic. Measuring only quantitative features of such systems is certainly possible but in many cases does not reflect a measurement of the claimed scientific contributions, since they are first and foremost of a qualitative nature.

To our knowledge, there is no clinical imaging database system that fuses image metadata (patient name, age, gender, etc.) and medical annotations (anatomy, imaging characteristic, disease) in the same formalism and allows searching for both in a unified manner. Thus we decided against presenting tables of retrieval times of our system 14 Firstly, because they do not really reflect the important features of this system and secondly because they are not comparable to existing systems in clinical practice. Instead we would like to discuss three query scenarios which contrast the features of RadSem with existing systems.

Scenario 1: "Give me all images of patient X." This can be handled directly by existing clinical image data base systems (PACS15) since this query can be

\footnotetext{
$\overline{13}$ The multilingual synonyms are not available for all FMA classes.

${ }^{14}$ Our efforts, e.g., for materialization of distances as described above, result in retrieval times always below one second even for large query expansions.

15 Picture Archiving and Communications Systems.
} 
reduced to a simple keyword search across metadata elements. RadSem supports this type of query in the same manner.

Scenario 2: "Give me all images from patients with a broken index finger." Existing systems require two tasks to be performed for the retrieval of this type of query. In the first task, the radiology information system (RIS) which stores all radiological findings has to be searched for textual descriptions of "broken index fingers". The facts that radiologists tend to use abbreviations in their reports and that the same medical incident could also be described using different words (e. g., fracture of index finger) or a different language limit the recall significantly. The second task for the radiologist is then to choose to retrieve a selection of images referenced by the radiology reports. Not all of these images necessarily have to deal with a "broken index finger" but might also deal with fractures of other bones which the patient might have had during the same time.

In RadSem both tasks can be performed in a single step. In the free text search, the query would simply be "anatomy:index disease:fracture".

Scenario 3: "Give me all pictures dealing with enlarged lymph nodes." Patients potentially suffering from lymph node cancer (lymphoma) are usually scanned using Computed Tomography, treated if cancerous lymph nodes are detected and called in for a number of follow-up examinations after the treatment over a period of several months. During these follow-up examinations the radiologist searches for lymph nodes which have changed in size compared to previous examinations. This requires access to previous images. For the same patient, this query can be handled by existing PACS. Their interfaces usually allow opening of any image series available for the current patient.

One important advantage of RadSem is that the radiologist here can also directly search for images from other patients with the clinical finding of having enlarged lymph nodes. Additionally, the query is not limited to a specific lymph node. The query "anatomy:lymph_node visual:enlarged" retrieves all images which are annotated with the anatomical concept "Lymph node" or any part of it and the visual characteristic enlarged. One could also think of refining this query by adding date ranges, patient gender, etc.

Summary: These examples clearly show that there are severe limitations for searching existing medical image database systems. Despite the fact that there are standardized vocabularies such as ICD-10 and well developed ontologies like the FMA, these technical developments have not yet found adaptation in clinical practice. This section pointed out these limitations and described our approach to overcome them by using a common representation for medical background knowledge and annotations, patient metadata and image acquisition parameters using the Semantic Web standards RDF and OWL.

\section{Conclusion and Future Work}

We have presented a semantic annotation and retrieval tool RadSem for medical images. It leverages on background information about the medical domain from 
formal ontologies and also covers clinical data management at several steps. Ontology based metadata extraction and medical annotations are represented using RDF and OWL and lay the basis for clearly arranged information visualizations of patient history, image annotations and search results. We presented different approaches to minimize the additional effort for adding medical annotations.

We have described our approach for formalizing the structure of the ICD-10 in OWL and presented several augmentations and mappings for FMA with further medical knowledge (e.g., about tissue types) and external data sources.

Articles about medicine in Wikipedia are in the majority of the cases understandable for a general audience but at the same time the scientific level of the articles is rather low compared to peer reviewed scientific publications as for example in PubMed 16 Therefore we will apply the technique described in Sect. 3.6 to generate links from ICD-10 concepts to relevant PubMed articles.

On the imaging side, we plan to integrate low-level content-based image retrieval techniques to additionally support query by image in conjunction with the presented search across medical annotations and patient metadata. We also aim to integrate fully-automatic semantic image parsing techniques to provide facilities for automatic image segmentation and the automatic suggestion of semantic image annotations.

\section{References}

1. Agne, S., Reuschling, C., Dengel, A.: DynaQ - dynamic queries for electronic document management. In: 10th IEEE International Enterprise Distributed Object Computing Conference Workshops (EDOCW 2006) (2006)

2. Bechhofer, S., Volz, R., Lord, P.W.: Cooking the semantic web with the OWL API. In: Fensel, D., Sycara, K.P., Mylopoulos, J. (eds.) ISWC 2003. LNCS, vol. 2870, pp. 659-675. Springer, Heidelberg (2003)

3. Bodenreider, O.: Biomedical ontologies in action: role in knowledge management, data integration and decision support. In: International Medical Informatics Association (IMIA) (ed.) IMIA Yearbook 2008, pp. 67-79. Schattauer (2008)

4. World Health Organization Geneva. International statistical classification of diseases and related health problems (2004)

5. Gruber, T.R.: Toward principles for the design of ontologies used for knowledge sharing. International Journal of Human-Computer Studies 43, 907-928 (1995)

6. Güld, M.O., Kohnen, M., Keysers, D., Schubert, H., Wein, B.B., Bredno, J., Lehmann, T.M.: Quality of DICOM header information for image categorization. Proc. SPIE 4685(39), 280-287 (2002)

7. Hayes, P.: RDF semantics. W3C Recommendation (February 2004)

8. Langlotz, C.P.: Radlex: A new method for indexing online educational materials. RadioGraphics 26, 1595-1597 (2006)

9. McGuinness, D.L., van Harmelen, F.: OWL Web Ontology Language overview. W3C recommendation, World Wide Web Consortium (February 2004)

10. Mejino, J.L., Rubin, D.L., Brinkley, J.F.: FMA-RadLex: An application ontology of radiological anatomy derived from the foundational model of anatomy reference ontology. In: AMIA Annual Symposium Proceedings, p. 465 (2008)

16 http://wWw.ncbi.nlm.nih.gov/pubmed 
11. Möller, M., Sintek, M.: A scalable architecture for cross-modal semantic annotation and retrieval. In: Dengel, A.R., Berns, K., Breuel, T.M. (eds.) KI 2008. LNCS, vol. 5243. Springer, Heidelberg (2008)

12. Möller, M., Sintek, M., Buitelaar, P., Mukherjee, S., Zhou, X.S., Freund, J.: Medical image understanding through the integration of cross-modal object recognition with formal domain knowledge. In: Proc. of HEALTHINF 2008, Funchal, Madeira, Portugal, vol. 1, pp. 134-141 (2008)

13. Mojsilovic, A., Gomes, J., Rogowitz, B.E.: Semantic-friendly indexing and querying of images based on the extraction of the objective semantic cues. International Journal of Computer Vision 56, 79-107 (2004)

14. Mueen, A., Zainuddin, R., Baba, M.S.: Automatic multilevel medical image annotation and retrieval. Journal of Digital Imaging 21(3), 290-295 (2008)

15. Mykowiecka, A., Marciniak, M., Podsiadly-Marczykowska, T.: Data-driven ontologies for an information extraction system from polish mammography reports. In: Proc. of the 10th International Protégé Conference, Budapest, Hungary (July 2007)

16. Pietriga, E., Bizer, C., Karger, D.R., Lee, R.: Fresnel: A browser-independent presentation vocabulary for RDF. In: Cruz, I., Decker, S., Allemang, D., Preist, C., Schwabe, D., Mika, P., Uschold, M., Aroyo, L.M. (eds.) ISWC 2006. LNCS, vol. 4273, pp. 158-171. Springer, Heidelberg (2006)

17. Prud'hommeaux, E., Seaborne, A.: SPARQL query language for RDF. Technical report, W3C (March 2007)

18. Raicu, D.S., Varutbangkul, E., Furst, J.D., Armato III, S.G.: Modeling Semantics from Image Data: Opportunities from LIDC. International Journal of Biomedical Engineering and Technology (2007)

19. Rosse, C., Mejino, R.L.V.: A reference ontology for bioinformatics: The foundational model of anatomy. Journal of Biomedical Informatics 36, 478-500 (2003)

20. Rubin, D.L., Mongkolwat, P., Kleper, V., Supekar, K., Channin, D.S.: Medical imaging on the semantic web: Annotation and image markup. In: AAAI Spring Symposium Series, Stanford University (2008)

21. Semy, S.K., Pulvermacher, M.K., Obrst, L.J.: Toward the use of an upper ontology for U.S. government and U.S. military domains: An evaluation. Technical report, MITRE Corporation (September 2004)

22. Temal, L., Dojat, M., Kassel, G., Gibaud, B.: Towards an ontology for sharing medical images and regions of interest in neuroimaging. Journal of Biomedical Informatics (2008)

23. Yao, J., Antani, S., Long, R., Thoma, G., Zhang, Z.: Automatic medical image annotation and retrieval using SECC. In: Proc. of 19th International Symposium on Computer-Based Medical Systems (CBMS 2006), Salt Lake City (June 2006) 